\title{
STAR FORMATION IN THE EXTENDED GASEOUS DISK OF THE ISOLATED GALAXY CIG 96
}

\author{
D. Espada ${ }^{1,2,3}$, J. C. Muñoz-Mateos ${ }^{4,5}$, A. Gil de Paz ${ }^{4}$, J. Sabater ${ }^{1,6}$, S. Boissier ${ }^{7}$, S. Verley ${ }^{8}$, E. Athanassoula ${ }^{7}$, \\ A. Bosma ${ }^{7}$, S. Leon ${ }^{9}$, L. Verdes-Montenegro ${ }^{1}$, M. Yun ${ }^{10}$, And J. Sulentic ${ }^{1}$ \\ ${ }^{1}$ Instituto de Astrofísica de Andalucía, CSIC, Apdo. 3004, 18080 Granada, Spain \\ ${ }^{2}$ National Astronomical Observatory of Japan, 2-21-1 Osawa, Mitaka, Tokyo 181-8588, Japan; daniel.espada@nao.ac.jp \\ ${ }^{3}$ Harvard-Smithsonian Center for Astrophysics, 60 Garden Street, Cambridge, MA 02138, USA; despada@cfa.harvard.edu \\ ${ }^{4}$ Departamento de Astrofísica y CC. de la Atmósfera, Universidad Complutense de Madrid, Avda. de la Complutense, s/n, E-28040 Madrid, Spain \\ ${ }^{5}$ National Radio Astronomy Observatory, 520 Edgemont Road, Charlottesville, VA 22903-2475, USA \\ ${ }^{6}$ Institute for Astronomy, University of Edinburgh, Edinburgh EH9 3HJ, UK \\ ${ }^{7}$ Laboratoire d'Astrophysique de Marseille, OAMP, Université Aix-Marseille \& CNRS UMR 6110, \\ 38 rue Frédéric Joliot-Curie, 13388 Marseille Cedex 13, France \\ ${ }^{8}$ Dept. de Física Teórica y del Cosmos, Universidad de Granada, Granada, Spain \\ ${ }^{9}$ Joint ALMA Observatory/ESO, Av. Alonso de Cordova 3107, Vitacura, Santiago, Chile \\ ${ }^{10}$ Department of Astronomy, University of Massachusetts, Amherst, MA 01003, USA \\ Received 2010 September 11; accepted 2011 April 25; published 2011 June 30
}

\begin{abstract}
We study the Kennicutt-Schmidt star formation law and efficiency in the gaseous disk of the isolated galaxy CIG 96 (NGC 864), with special emphasis on its unusually large atomic gas $(\mathrm{HI})$ disk $\left(r_{\mathrm{HI}} / r_{25}=3.5, r_{25}=1\right.$ '.85). We present deep Galaxy Evolution Explorer near- and far-UV observations, used as a recent star formation tracer, and we compare them with new, high-resolution $\left(16^{\prime \prime}\right.$ or $\left.1.6 \mathrm{kpc}\right)$ Very Large Array H i observations. The UV and H I maps show good spatial correlation outside the inner $1^{\prime}$, where the $\mathrm{H}$ I phase dominates over $\mathrm{H}_{2}$. Star-forming regions in the extended gaseous disk are mainly located along the enhanced H I emission within two (relatively) symmetric, giant gaseous spiral arm-like features, which emulate an $\mathrm{H}$ i pseudo-ring at $r \simeq 3^{\prime}$. Inside this structure, two smaller gaseous spiral arms extend from the northeast and southwest of the optical disk and connect to the previously mentioned $\mathrm{H}_{\mathrm{I}}$ pseudo-ring. Interestingly, we find that the (atomic) Kennicutt-Schmidt power-law index systematically decreases with radius, from $N \simeq 3.0 \pm 0.3$ in the inner disk $(0.8-1.7)$ to $N=1.6 \pm 0.5$ in the outskirts of the gaseous disk (3'3-4!2). Although the star formation efficiency (SFE), the star formation rate per unit of gas, decreases with radius where the $\mathrm{H}$ I component dominates as is common in galaxies, we find that there is a break of the correlation at $r=1.5 r_{25}$. At radii $1.5 r_{25}<r<3.5 r_{25}$, mostly within the $\mathrm{H}_{\text {I }}$ pseudo-ring structure, regions exist whose $\mathrm{SFE}$ remains nearly constant, $\mathrm{SFE} \simeq 10^{-11} \mathrm{yr}^{-1}$. We discuss possible mechanisms that might be triggering the star formation in the outskirts of this galaxy, and we suggest that the constant SFE for such large radii $\left(r>2 r_{25}\right)$ and at such low surface densities might be a common characteristic in extended UV disk galaxies.
\end{abstract}

Key words: galaxies: individual (NGC 864) - galaxies: ISM - galaxies: spiral - galaxies: structure - stars: formation

Online-only material: color figures

\section{INTRODUCTION}

For three decades it has been known that about one-third of galaxies show unusually extended atomic gas $\left(\mathrm{H}_{\mathrm{I}}\right)$ distributions (e.g., Bosma 1981; Huchtmeier \& Richter 1982). It was not until recently, with the advent of the Galaxy Evolution Explorer (GALEX; Martin et al. 2005), that we could easily observe star formation (SF) far beyond the optical radius of galaxies (Thilker et al. 2005, 2007, 2009; Gil de Paz et al. 2005, 2007; Boissier et al. 2007). The star-forming regions generally show a good spatial correlation with the H I component (the main phase of neutral gas at large radii) in these extended $\mathrm{H}$ I disks (e.g., Bigiel et al. 2010b).

These extended ultraviolet (XUV) disks are characterized by very blue UV-optical colors, they can reach up to four times the optical radius (as given by the $\mu_{B}=25 \mathrm{mag} \operatorname{arcsec}^{-2}$ major axis isophote), and are mainly composed of low-mass stellar clusters of $10^{3}-10^{6} M_{\odot}$. Recent studies have shown the presence of moderate amounts of dust and oxygen abundances of $Z_{\odot} / 10$ (Gil de Paz et al. 2007; Dong et al. 2008; Bresolin et al. 2009). Thilker et al. (2007) recently classified the XUV disks into Type 1 (patchy UV emission beyond the canonical SF threshold; Toomre 1964; Martin \& Kennicutt 2001) and Type 2 (blue, large compared with the size of the galaxy in the nearIR disks inside the SF threshold) and found XUV disks to be relatively frequent, $20 \%$ and $10 \%$ of the overall disk-galaxy population, for Types 1 and 2, respectively.

Comparing UV and $\mathrm{H} \mathrm{I}$ in these extended disks allows the study of the Kennicutt-Schmidt (KS) law, or star formation rate (SFR) versus gas surface densities, $\Sigma_{\mathrm{SFR}}$ versus $\Sigma_{\text {gas }}$ (Schmidt 1959; Kennicutt 1998), in the extreme low-density and often low-metallicity environments in the outskirts of galaxy disks, which can elucidate the limiting conditions for gas cloud formation and SF (Toomre 1964; Martin \& Kennicutt 2001). In addition, it is essential to study the relation between the availability of $\mathrm{H}$ i in the outer disk and the depletion timescales due to SF. Such an Hi reservoir should play a role in the replenishment of the molecular gas content to enable future SF. This process can thus contribute to sustain SF over cosmological times (e.g., Kennicutt et al. 1994; Bauermeister et al. 2010).

Another key question to be addressed is what are the mechanisms for triggering SF in these low-density gaseous environments. Several mechanisms have been proposed, both of external and internal origin, including gravitational instabilities 
induced by galaxy interactions (e.g., Gil de Paz et al. 2005) or the impact of dark matter subhalos or extragalactic clouds (TenorioTagle 1981; Bekki \& Chiba 2006), turbulence compression, supernovae, or gaseous spiral arms (Elmegreen \& Hunter 2006). In this regard, Thilker et al. (2007) found that $\sim 75 \%$ of the Type 1 XUV-disk objects show some kind of optical morphology peculiarity or $\mathrm{H}$ I evidence for interaction/merger or a minor external perturbation such as primordial clouds (Thilker et al. 2009). For instance, NGC 4262, a member of the Virgo cluster, exhibits an extended $\mathrm{H}$ I and UV ring-like feature probably formed as a result of a past major interaction episode (Bettoni et al. 2010).

Thilker et al. (2007) noted that isolated galaxies might also host a Type 1 XUV disk. Although studying such isolated systems can provide information about other mechanisms producing intrinsic extended disks, so far no detailed study has been performed. An excellent target sample is the Catalog of Isolated Galaxies (CIG; Karachentseva 1973). The isolation of the galaxies in the catalog has been revised within the AMIGA project (Analysis of the interstellar Medium of Isolated Galaxies, http://amiga.iaa.es; Verdes-Montenegro et al. 2005; Verley et al. 2007a, 2007b). The imposed isolation criterion ensures that the galaxies have not suffered any major interactions in a timescale of the order of 3 Gyr (Verdes-Montenegro et al. 2005). We searched the GALEX Nearby Galaxy Survey (NGS; Gil de Paz et al. 2007), Deep Imaging Survey (DIS), Medium Imaging Survey (MIS), and All Sky Survey (AIS) for galaxies in the AMIGA sample. Out of the 24 galaxies in the AMIGA sample having MIS, DIS, and/or NGS data, none showed evidence of XUV emission. We found a total of 126 objects in AIS. Out of these, we identified seven XUV-disk galaxies, four of them being Type 1 (CIG 96, CIG 103, CIG 712, and CIG 812).

In this paper, we examine the properties of the extended disk in the SAB(rs)c (de Vaucouleurs et al. 1991) galaxy CIG 96 (NGC 864; Tully 1988). CIG 96 has a strong and slightly curved bar, with a bar length estimated by Fourier analysis in the $H$ band of 26" (Eskridge et al. 2002; Speltincx et al. 2008). It is located nearby at a distance of $17.3 \mathrm{Mpc}\left(V=1561.6 \mathrm{~km} \mathrm{~s}^{-1}\right.$; Espada et al. 2005, using an $H_{0}=75 \mathrm{~km} \mathrm{~s}^{-1} \mathrm{Mpc}^{-1}$ ), which yields a linear scale of $1^{\prime \prime} \simeq 100 \mathrm{pc}$. We adopted for CIG 96 the majorand minor-axis optical diameters $D_{25}=3$ '. 7 and $d_{25}=2$ '. 6 at the isophotal level $25 \mathrm{mag} \operatorname{arcsec}^{-2}$ in the $B$ band (homogenized value from LEDA; Paturel et al. 2003). Note that the diameters in the NASA/IPAC Extragalactic Database are larger, $D_{25}=$ 4.7 and $d_{25}=3.5$, as in the Uppsala General Catalogue of Galaxies (Nilson 1973). However, the homogenized diameters given by LEDA are consistent with recent values found in the literature, such as Paturel et al. (2000). We assume throughout the paper a position angle (P.A.) $=23^{\circ} \pm 3^{\circ}$ and an inclination $i=43^{\circ} \pm 2^{\circ}$, values derived from modeling of the $\mathrm{H}$ I data cube in Espada et al. (2005). These are also consistent to the homogenized values presented in LEDA, P.A. $=24.0$ and $i=46.7$.

Very Large Array (VLA) H I maps with resolutions of about $\sim 45^{\prime \prime}$ revealed an unusually extended $\mathrm{H}$ i disk (Espada et al. 2005). Besides the optical revision of the isolation (Verley et al. 2007a), CIG 96 is also found to be isolated from any relatively H I-rich galaxy within the VLA primary beam $\left(\sim 30^{\prime}\right)$. Only a diminutive galaxy with an $\mathrm{H}_{\mathrm{I}}$ mass $M_{\mathrm{HI}} \simeq 5 \times 10^{6} M_{\odot}$ located at $80 \mathrm{kpc}$ from CIG 96 is its nearest companion. No tidal tails or other signs of interaction were found to the reached sensitivity. Largest $\mathrm{H}$ I column densities were found within a ring feature with major and minor axes of $1.5 \times 11^{\prime} .0$. More interestingly, Espada et al. (2005) found a pseudo-ring feature with an approximate size of $8.2 \times 44^{\prime} 6$. The galaxy $\mathrm{H}_{\text {I disk has }}$ a kinematic asymmetry. The rotation curves in the approaching and receding sides differ, mostly as a result of a kinematically detached clump, which is evident as a secondary peak in the position velocity cut at radii $r \sim 6.5$ to the southwest (SW; Espada et al. 2005).

The main goal of this paper is to shed light on the local KS laws and star formation efficiency (SFE), the SFR per unit of gas, of the extended gaseous component in this isolated object, using new deep GALEX observations and high-resolution $\left(\sim 15^{\prime \prime}\right)$ VLA H I observations (Section 2). In Section 3 we describe our Hi and UV maps, compare the spatial location of UV relative to $\mathrm{HI}$, and study the KS law locally for different radius ranges as well as the local SFE as a function of radius. In Section 4 we compare the observed $\Sigma_{\text {gas }}$ and rotation curve with that obtained from a model fitting using optical, UV, and IR photometric radial profiles. Finally, we provide a discussion on the main findings and our conclusions in Section 5.

\section{OBSERVATIONS AND DATA REDUCTION}

\subsection{VLA H I Data}

H I observations were carried out with the NRAO VLA ${ }^{11}$ in its $\mathrm{C}$ configuration on 2005 July 23, and were combined to the D configuration data published by Espada et al. (2005). We used the same correlator setting as for the D configuration data, which provided a velocity resolution of $48.8 \mathrm{kHz}\left(10.4 \mathrm{~km} \mathrm{~s}^{-1}\right)$ for the 64 individual channels after Hanning smoothing. The editing and calibration of the data were done with AIPS. ${ }^{12}$ The data were imaged using IMAGR by combining both data sets. For the cleaning process, we applied a compromise between natural and uniform weighting (weighting parameter ROBUST $=0$ ). The average of the line-free channels has been subtracted from all the individual channels.

The final rms noise level achieved after 4 (D configuration) plus $7 \mathrm{hr}$ (C configuration) per channel is $\sigma=0.4 \mathrm{mJy} \mathrm{beam}^{-1}$, with a synthesized beam of $\sim 16^{\prime \prime} .9 \times 15^{\prime \prime} .6$ (or $1.7 \times 1.6 \mathrm{kpc}$ ), and P.A. $=-30.10$. We obtained the integrated intensity (moment 0 ) map from the 27 channels with $\mathrm{H}$ I emission, to which we applied a primary beam correction (HPBW $\sim 30^{\prime}$ for the VLA antennas). The moment 0 was masked using a $3 \sigma\left(=1.2 \mathrm{mJy}^{\text {beam }}{ }^{-1}\right)$ clipping for each single channel. The $1 \sigma$ noise level of the moment 0 map is $0.02 \mathrm{Jy} \mathrm{beam}^{-1} \mathrm{~km} \mathrm{~s}^{-1}$, calculated as the addition in quadrature of the channel's $1 \sigma$ levels.

The excellent agreement between the single-dish total flux, $S=102.96 \mathrm{Jy} \mathrm{km} \mathrm{s}^{-1}$ using the Green Bank $43 \mathrm{~m}$ Telescope (HPBW $=22^{\prime}$; Haynes et al. 1998), and that obtained with the VLA C+D configuration data, $S=100.3 \mathrm{Jy} \mathrm{km} \mathrm{s}^{-1}$, implies that we recover most of the flux. Thus, an extended gas component is not expected to be missing in our maps.

\subsection{GALEX Near-ultraviolet and Far-ultraviolet Data}

The GALEX observatory provides both a near-UV (NUV $\lambda=1771-2831 \AA$ ) and a far-UV (FUV $\lambda=1344-1786 \AA$ ) broadband filter, with angular resolutions (FWHM) of 5.3 and 4 ". 2 , respectively. The field of view of the instrument is about 1.25 , much larger than the VLA primary beam. CIG 96 was observed in NUV for $6.385 \mathrm{ks}$ and in FUV for $1.648 \mathrm{ks}$ in

\footnotetext{
11 The National Radio Astronomy Observatory is a facility of the National Science Foundation operated under cooperative agreement by Associated Universities, Inc.

12 http://www.aips.nrao.edu/cook.html
} 

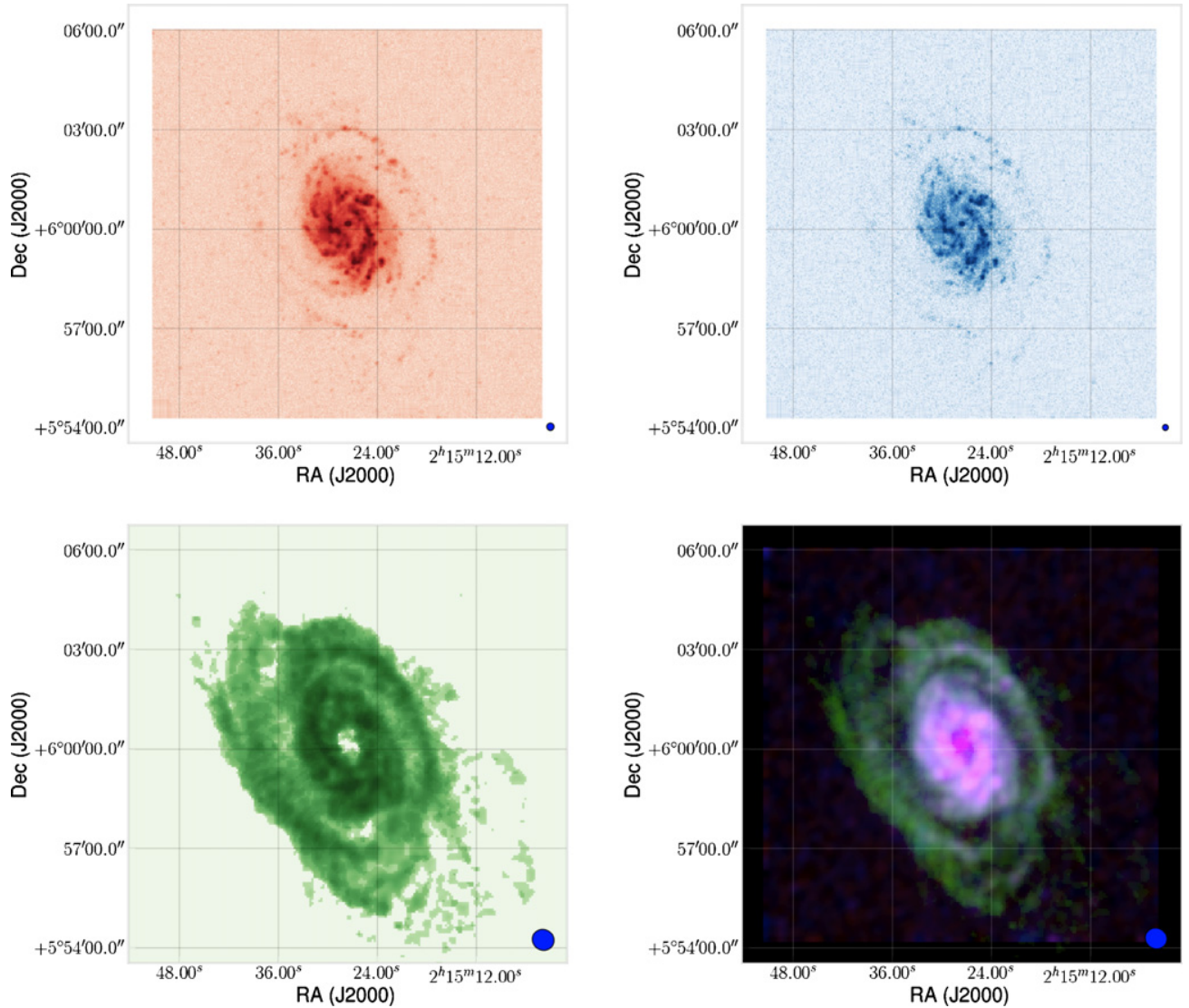

Figure 1. GALEX NUV (upper left, red) and FUV (upper right, blue) maps, as well as the VLA (C+D configuration) H I moment 0 map of CIG 96 (lower left, green), and a composite of the three maps (lower right) where the resolution of the UV maps have been degraded to that of the H I. All the panels cover a field of view of $13^{\prime} \times$ $13^{\prime}$. The resolution of each map is shown in the lower right.

our GALEX Guest Investigator program 065 in Cycle 5. We also combined these data with previous observations from the archive.

Foreground stars are prominent in the NUV images and we identified and flagged them easily via their UV colors (see Gil de Paz et al. 2007). We computed and subtracted the background from both the FUV and NUV maps. We corrected fluxes by adopting Galactic extinction as given by Schlegel et al. (1998), namely $E(B-V)=0.048$.

Besides correcting for the foreground Galactic extinction, we must also account for radial variations in the internal extinction. The total IR (TIR) to UV ratio (TIR/UV) is a robust tracer of internal extinction, depending weakly on the dust-to-stars geometry or the extinction law (see, e.g., Witt \& Gordon 2000; Buat et al. 2005, and references therein). In order to compute the TIR radial profiles, Spitzer images at 24, 70, and $160 \mu \mathrm{m}$ would be required, but they are not available for this galaxy. Therefore, we resorted to the FUV-NUV color (i.e., the UV slope) as an indirect tracer of dust extinction. Instead of using the classical calibrations derived for starburst galaxies (Calzetti et al. 1994; Heckman et al. 1995; Meurer et al. 1999), here we made use of the recipes of Muñoz-Mateos et al. (2009), which were calibrated on a sample of normal, nearby galaxies. From the FUV-NUV color profile we infer the TIR/FUV and TIR/NUV at each radius. These ratios are then converted into extinction radial profiles in the FUV and NUV bands independently. This was done by means of the prescriptions of Cortese et al. (2008), which take into account the varying contribution of young and old stars to the dust heating. The error in the extinction is dominated by the scatter of ratios when we calibrate TIR/
FUV and TIR/NUV as a function of color (FUV-NUV) and is estimated to be of the order of $0.4 \mathrm{mag}$.

\section{RESULTS}

\subsection{H I Component}

We present the $\mathrm{H}_{\mathrm{I}}$ integrated density distribution in Figure 1 (lower left panel, green). The $\mathrm{H}$ I extent is $D_{\mathrm{HI}}=12.9$ at a level of $0.7 M_{\odot} \mathrm{pc}^{-2}$ (1 $\sigma$ noise level, Section 2.1), which results in an unusually large $\mathrm{H}$ I to optical extent ratio of $D_{\mathrm{HI}} / D_{25} \simeq 3.5$. A similar ratio is that of M83, whose XUV-disk properties have been previously studied in detail (Thilker et al. 2005, 2007; Bigiel et al. 2010a).

The VLA C+D configuration H I map reveals a large variety of structures that could not be clearly distinguished in the VLA D-configuration map due to the lower angular resolution. The inner ring, with projected diameters of $1.5 \times 1.0$, exhibits enhanced $\mathrm{HI}$ emission along the stellar spiral arms. The outer pseudo-ring, with an extent of $8.2 \times 4.6$, seems to be connected to the inner ring to the northeast (NE) and SW of the inner disk. Overall, the shape of the outer pseudo-ring resembles two giant spiral arms surrounding the optical disk.

Although the shape of the brighter features is quite symmetric, there is also a faint diffuse $\mathrm{H}$ I emission that is asymmetric. The kinematically detached component in the SW that was reported in Espada et al. (2005) at about a $6^{\prime}$ radius is seen here as a clumpy and extended component. A more detailed analysis of the entire $\mathrm{H}$ I data cube will be presented in a forthcoming paper. 


\subsection{FUV and NUV Emission}

We present the NUV map (upper left panel, red) and FUV map (upper right panel, blue) in Figure 1 with the same field of view as the Hi map. Both NUV and FUV maps show an almost identical distribution. However, while the radially averaged FUV-NUV color is $\sim 0.5$ in the internal parts of the disk (in units of $\mathrm{AB}$ magnitudes per square arcsec), it reaches $\mathrm{FUV}-\mathrm{NUV}=0.1-0.2$ in the most external regions.

First, there are two nearly symmetric arms, one starting at the $\mathrm{E}$ of the bar and extending toward the $\mathrm{N}$, and another one starting at the $\mathrm{W}$ of the bar and extending toward the S. The SW arm's appearance in both NUV and FUV emission is clumpy. This is similar in appearance to that observed by Eskridge et al. (2002) in the optical and near-IR (NIR). Eskridge et al. claim that the spiral pattern is flocculent in the outer parts of the inner optical/NIR disk $(r<1$ 1.5). These flocculent regions are related to the connections between the outer Hi pseudo-ring and the inner $\mathrm{H}$ I ring to the $\mathrm{NE}$ and $\mathrm{SW}$ at the P.A. of the inner disk. A central concentration is found unresolved, which is likely connected to a nuclear starburst (Martini et al. 2003).

Second, a more diffuse and extended component of UV emission is seen in the UV maps. Two symmetric spiral arms are apparent, extending from the NE and SW of the optical disk. These spiral arms join an outer pseudo-ring structure to the $\mathrm{E}$ and $\mathrm{W}$ starting at about $r=2^{\prime}$. The pseudo-ring structure is the same as that seen in the $\mathrm{H}$ i outer disk, although the star-forming regions seem to be more confined to regions with high $\mathrm{H}$ i surface density. Other filamentary and more chaotic structures are seen out to almost $r=6^{\prime}$, especially to the SW and to a minor extent in the NE.

A composite image of the $\mathrm{H}$ I and NUV/FUV maps convolved to the resolution of the former is presented in Figure 1 (lower right panel). A scheme (over the composite image) of main features in the outskirts of CIG 96 is shown in the upper panel of Figure 2: spiral arms (blue crosses) extending from the $\mathrm{N}$ to the $\mathrm{W}$ and from the $\mathrm{S}$ to the $\mathrm{E}$, connecting the pseudo-ring structure (red crosses). The circles indicate the southeast (SE) spiral arm and pseudo-ring (SE side as well) rotated by $180^{\circ}$, which shows that these features are nearly symmetrical.

The lower panel of Figure 2 shows that the NUV (color pixel map) and Hi maps (contour map) have a high degree of correlation. This tight spatial correlation of $\mathrm{H}$ I and UV emission remains far into the outer pseudo-ring and a diffuse component all over the $\mathrm{H}_{\mathrm{I}}$ envelope, as can be seen in the last plotted contour.

\subsection{Star Formation Law}

We obtained the KS law (Schmidt 1959; Kennicutt 1998) pixel-by-pixel by comparing the gas surface density $\left(\Sigma_{\text {gas }}\right)$ map obtained from our H I data (Section 2.1) and the SFR surface density $\left(\Sigma_{\mathrm{SFR}}\right)$ from the extinction-corrected (radially) NUV data (Section 2.2). We converted the pixel size of both maps to $16^{\prime \prime}$ (as the H I beam size).

In the outskirts of disks the gaseous component is usually dominated by $\mathrm{H} \mathrm{I}$ rather than by molecular gas, so the $\mathrm{H}$ I content is a good estimate of the total gas content, $\Sigma_{\text {gas }} \simeq \Sigma_{\mathrm{HI}}$, especially below the saturation limit between atomic and molecular gas at $\Sigma_{\text {gas }} \simeq 12 M_{\odot} \mathrm{pc}^{-2}$ (Martin \& Kennicutt 2001; Wong \& Blitz 2002; Bigiel et al. 2008; Verley et al. 2009, 2010).

In order to calculate the $\Sigma_{\text {gas }}$, we took into account the inclination of the galaxy and multiplied by 1.36 to include the
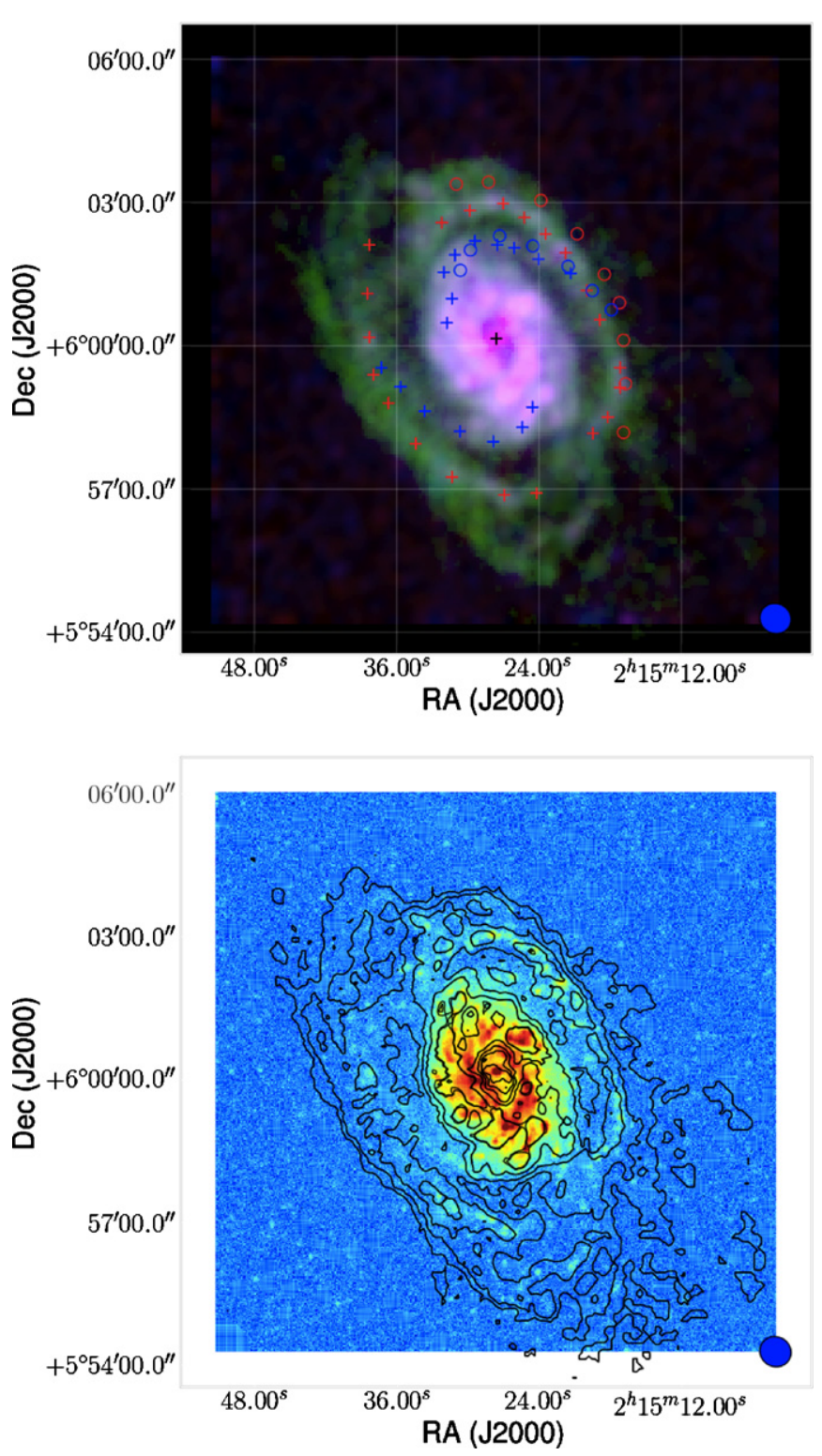

Figure 2. Upper panel: scheme (over the composite image) of the gaseous spiral arms (blue crosses) extending from the $\mathrm{N}$ to the $\mathrm{W}$ and from the $\mathrm{S}$ to the $\mathrm{E}$, and the pseudo-ring structure (red crosses). The circles indicate the SE spiral arm and the SE pseudo-ring side, rotated by $180^{\circ}$. Lower panel: H I map in contours over the NUV color (logarithmic) scale map. The H I contours are $0.02,0.08,0.16,0.24$, and $0.32 \mathrm{Jy}_{\text {beam }}{ }^{-1} \mathrm{~km} \mathrm{~s}^{-1}$, or $\Sigma_{\mathrm{HI}}=0.5,1.9,3.9,5.8$, and $7.8 M_{\odot} \mathrm{pc}^{-2}$.

helium contribution (Walter et al. 2008; Leroy et al. 2008):

$$
\Sigma_{\mathrm{gas}}\left[M_{\odot} \mathrm{pc}^{-2}\right]=33.6 \times \Sigma_{i} \mathrm{~S}_{i}\left[\mathrm{Jy} \mathrm{beam}^{-1}\right] \Delta V\left[\mathrm{~km} \mathrm{~s}^{-1}\right],
$$

where $S_{i}$ is the flux density for each channel and $\Delta V$ is the channel velocity width. The $1 \sigma$ noise level for $\Sigma_{\text {gas }}=$ $0.7 M_{\odot} \mathrm{pc}^{-2}$, using the sensitivity limit reached in our $\mathrm{HI}$ moment 0 map (Section 2.1).

As for the $\Sigma_{\mathrm{SFR}}$ map, we used the corrected NUV data (Section 2.2). In the literature, FUV is used more widely in order to derive SFR as it involves emission from young stars born in the last $100 \mathrm{Myr}$, more similar to the $\mathrm{H} \alpha(<10 \mathrm{Myr})$ than to the NUV emission (<300 Myr; Verley et al. 2009). However, by using NUV, we achieve a gain in sensitivity and NUV emission is not essentially different from FUV to our resolution. This approach can also be useful for other sources 
Table 1

Local Kennicutt-Schmidt Law for Different Annuli

\begin{tabular}{lccccc}
\hline \hline Zone & Radius Range $\left(^{\prime}\right) /(\mathrm{kpc})$ & $\begin{array}{c}\text { OLS(Y-X) } \\
\text { Slope }(N)\end{array}$ & Intercept & $\begin{array}{c}\text { Bisector } \\
\text { Slope }(N)\end{array}$ & Intercept \\
\hline 1 & $0.8-1.7(4.8-10.2)$ & $1.7 \pm 0.3$ & $-4.0 \pm 0.3$ & $3.0 \pm 0.3$ & $-5.4 \pm 0.3$ \\
2 & $1.7-2.5(10.2-15.0)$ & $1.9 \pm 0.3$ & $-4.9 \pm 0.2$ & $2.18 \pm 0.10$ & $-5.07 \pm 0.11$ \\
3 & $2.5-3.3(15.0-19.8)$ & $1.4 \pm 0.2$ & $-4.85 \pm 0.14$ & $1.7 \pm 0.2$ & $-5.1 \pm 0.3$ \\
4 & $3.3-4.2(19.8-25.2)$ & $1.2 \pm 0.2$ & $-5.1 \pm 0.10$ & $1.6 \pm 0.5$ & $-5.3 \pm 0.6$ \\
\hline
\end{tabular}

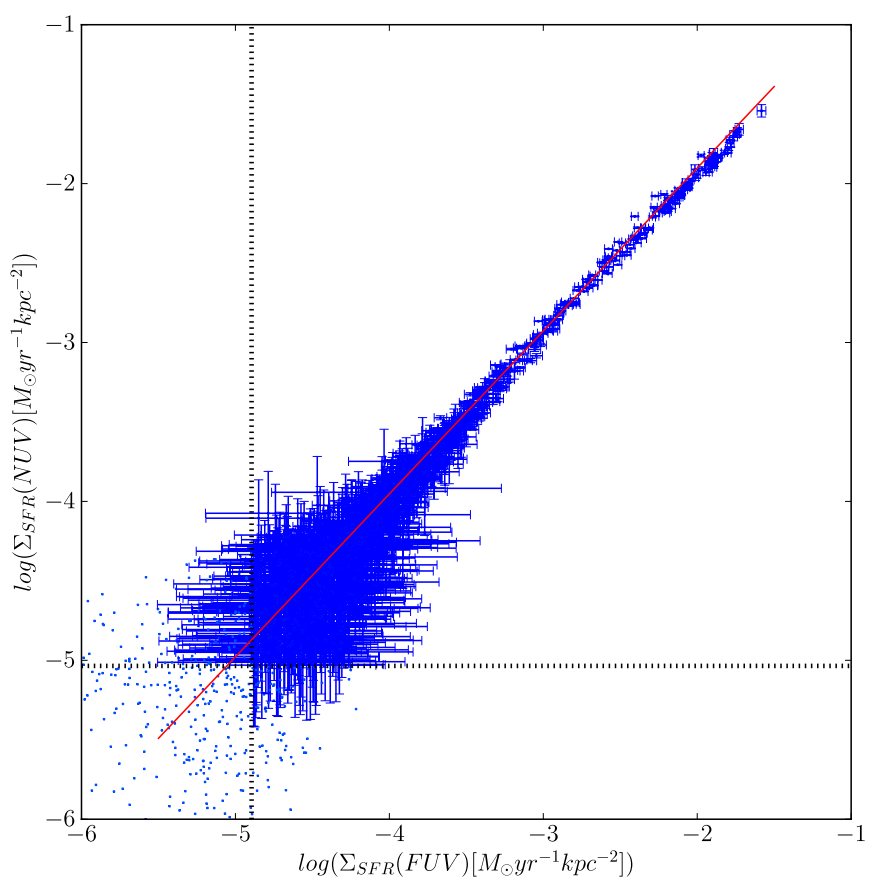

Figure 3. Pixel-to-pixel $\left(16^{\prime \prime}\right)$ comparison of SFR obtained from both extinctioncorrected NUV and FUV maps. The red line indicates the bisector fit using all the data points above the sensitivity limits, with a slope equal to unity (1.02 \pm $0.03)$ and an intercept close to $0(0.15 \pm 0.01)$. The two dashed lines represent the noise level limit for the SFR derived from NUV (horizontal) and FUV (vertical). Error bars have been calculated as explained in Section 3.3.

(A color version of this figure is available in the online journal.)

with more sensitive NUV data than FUV. As for the "hidden SF," i.e., the SF that we cannot correct from the UV color because it is completely obscured by dust in both bands, it is expected to be negligible in the outer parts of disks, since Prescott \& Kennicutt (2008) showed that there are no such regions at radii $r>0.6 r_{25}$ for SINGS galaxies (Kennicutt et al. 2003).

We convolved the NUV map to match the resolution of the H I map. Following Kennicutt (1998), the $\Sigma_{\text {SFR }}$ was calculated as

$$
\Sigma_{\mathrm{SFR}}\left[M_{\odot} \mathrm{yr}^{-1} \mathrm{kpc}^{-2}\right]=10^{-0.4 \times \mu_{\mathrm{NUV}}\left[\mathrm{AB} \operatorname{mag} \operatorname{arcsec}^{-2}\right]+7.41}
$$

assuming solar metallicity, a Salpeter (1955) initial mass function (IMF), and that the SFR has remained constant over the last few $10^{8} \mathrm{yr}$, which is the typical lifetime of stars dominating the $\mathrm{UV}$ emission. In this equation, $\mu_{\mathrm{NUV}}$ is the surface brightness (in AB magnitude units) of NUV emission. To convert to the SFR derived using the Kroupa (2001) IMF, one should multiply our SFR by a factor of 0.83 . The $1 \sigma$ sensitivity limit is $\Sigma_{\mathrm{SFR}}=$ $9.2 \times 10^{-6} M_{\odot} \mathrm{yr}^{-1} \mathrm{kpc}^{-2}$. In order to avoid projection effects, we have also deprojected both the $\Sigma_{\mathrm{SFR}}$ and the $\Sigma_{\text {gas }}$ maps.

Figure 3 shows the correlation between the $\Sigma_{\text {SFR }}$ derived from both the NUV and FUV map pixels (16" size) within the inner $400^{\prime \prime}$. The $\Sigma_{\mathrm{SFR}}$ obtained from the FUV map has been calculated in a similar manner to the $\Sigma_{\mathrm{SFR}}$ from the NUV map. The error bars presented in Figure 3 represent the formal uncertainties obtained from the sensitivity limit as well as from the uncertainties associated with the extinction correction. A bisector fit to all the data points yields a slope equal to unity and an intercept close to $0(1.02 \pm 0.03$ and $0.15 \pm 0.01$, respectively). We estimated the uncertainties using different random representations of the data taking into account the uncertainties (bootstrapping). We obtained similar uncertainties for the formal slope and intercept, 0.01 and 0.02 , respectively. Finally, note that calculations of $\Sigma_{\text {SFR }}$ using alternate SFR tracers give differences of the order of $50 \%$ (Bigiel et al. 2010b; Leroy et al. 2008; Verley et al. 2009). We do not consider this uncertainty in our error estimation.

In Figure 4 we show the $\Sigma_{\text {SFR }}$ versus $\Sigma_{\text {gas }}$ plot for CIG 96. From the figure we can discern the saturation limit between atomic and molecular gas found for other galaxies at $\Sigma_{\text {gas }} \simeq$ $12 M_{\odot} \mathrm{pc}^{-2}$.

In order to inspect whether the local KS SF law changes as a function of radius, we calculated a linear fit for different annuli centered at $\alpha(2000)=02^{\mathrm{h}} 15^{\mathrm{m}} 27^{\prime \prime} .64, \delta(2000)=06^{\circ} 00^{\prime} 09^{\prime \prime} .1$ (Leon \& Verdes-Montenegro 2003). Each color in the data points of the $\Sigma_{\text {SFR }}$ versus $\Sigma_{\text {gas }}$ plot in the lower panel of Figure 4 represents a different annulus zone over the deprojected H I map, which is depicted in the upper panel of Figure 4.

First, in the innermost region $\left(r<0^{\prime} .8\right.$ or $4.8 \mathrm{kpc}$; in dark blue in the left upper panel of Figure 4), the correlation between $\Sigma_{\text {SFR }}$ versus $\Sigma_{\text {gas }}$ is biased since at such radii the contribution of gas in molecular phase is presumably larger than that in atomic phase. For instance, $M_{\mathrm{H}_{2}}=2 \times 10^{8} M_{\odot}$ using $\mathrm{CO}(1-0)$ observation at the Swedish-ESO $15 \mathrm{~m}$ Submillimeter Telescope (SEST), which is characterized by an HPBW $=42^{\prime \prime}$ (radius $\sim 2 \mathrm{kpc}$; Elfhag et al. 1996; U. Lisenfeld et al. 2011, in preparation). If the molecular gas is distributed within a uniform distribution then the corresponding molecular gas surface density would be $\Sigma_{\mathrm{H}_{2}} \sim 11.5 M_{\odot} \mathrm{pc}^{-2}$. The $\Sigma_{\mathrm{H}_{2}}$ is likely larger since enhanced emission is expected toward the nucleus, inner spiral arms, and along the bar. Should we include the molecular gas contribution, the correlation $\Sigma_{\mathrm{SFR}}-\Sigma_{\text {gas }}$ would likely continue above $\Sigma_{\text {gas }}>$ $12 M_{\odot} \mathrm{pc}^{-2}$ in a standard manner, with a slope of $N \sim 1-1.4$ as it is observed in other galaxies (e.g., Bigiel et al. 2008).

We find that the power index (bisector fit) of the $\mathrm{KS}$ law systematically changes with radius from $N=3.0 \pm 0.3$ in the inner disk $(0.8-1.7$ or $4.8-10.2 \mathrm{kpc})$ to $N=1.6 \pm 0.5 \mathrm{in}$ the pseudo-ring feature (3'3-4.2 or $19.8-25.2 \mathrm{kpc})$. Further out the correlations are biased since a considerably large amount of data points fall below the sensitivity limit. We detailed in Table 1 the parameters for the fit with $\Sigma_{\text {gas }}$ as an independent variable (OLS $(\mathrm{Y}-\mathrm{X})$ ) as well as the least-square bisector fit, and for annuli from $0.8-4{ }^{\prime} .2$ in bins of 0.8 (4.8-25.2 $\mathrm{kpc}$ in bins of $4.8 \mathrm{kpc}$ ). The estimated errors of the fit parameters have been calculated via bootstrapping, in the same manner as in the $\Sigma_{\mathrm{SFR}}$ 

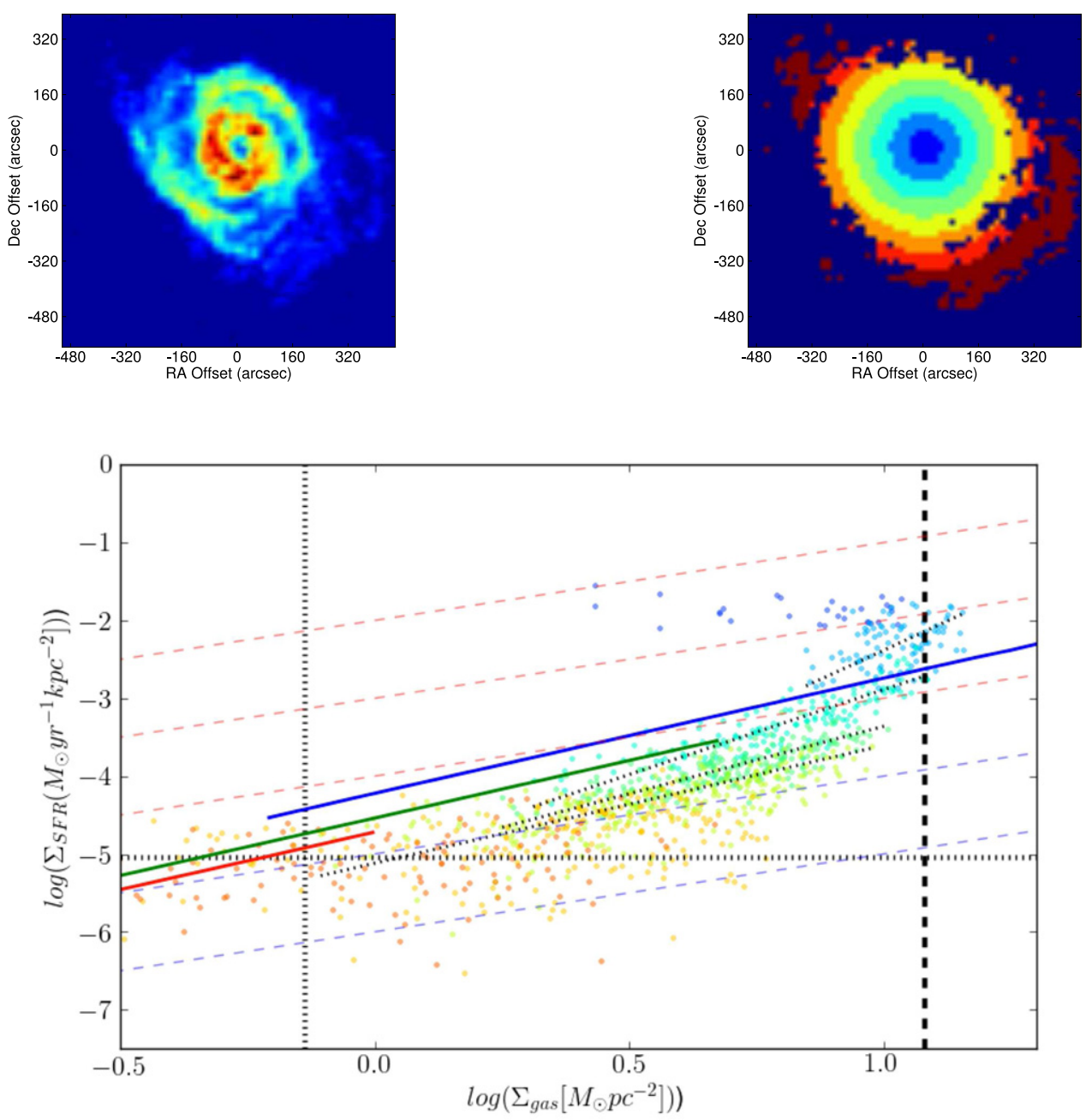

Figure 4. Upper panels: deprojected H I map (left), with the color scale showing different radial regions (right). Lower panel: $\Sigma_{\text {SFR }}$ vs. $\Sigma_{\text {gas }}($ KS law) pixel-to-pixel for the different rings. Linear fits (bisector) to KS law for the different regions are also shown as dotted lines (see Table 1). The diagonal (red and blue) dashed lines indicate constant SFE levels at $10^{-12}$ (bottom line), $10^{-11}, 10^{-10}, 10^{-9}$, and $10^{-8}$ (upper line) $\mathrm{yr}^{-1}$. The vertical thick dashed line shows the threshold between molecular and atomic gas at $\Sigma_{\mathrm{gas}}=12 M_{\odot} \mathrm{pc}^{-2}$. The vertical and horizontal thick dotted lines indicate the noise levels of $\Sigma_{\text {gas }}$ (vertical) and $\Sigma_{\mathrm{SFR}}$ (horizontal), at $0.7 M_{\odot} \mathrm{pc}^{-2}$ and $1.9 \times 10^{-6} M_{\odot} \mathrm{yr}^{-1} \mathrm{kpc}^{-2}$, respectively. The crosses represent the data points in the kinematically detached region in the SW (Espada et al. 2005 ).

comparison from NUV and FUV. Note that in general these parameters are not strongly affected due to spatial resolution. Bigiel et al. (2008) show that the power-law parameters vary only weakly with changing spatial resolution.

Besides the uncertainty that results from the assumption $\Sigma_{\text {gas }} \simeq \Sigma_{\mathrm{HI}}$, note that there is another source of uncertainty regarding a possible metallicity gradient that might affect the calculated $\Sigma_{\mathrm{SFR}}$ (in the sense that metallicity is lower in the outskirts). Assuming a constant calibration for a given metallicity, it can be inferred that regions with a lower metallicity would have a lower $\Sigma_{\text {SFR }}$ for a fixed UV surface brightness (Leitherer et al. 1999).

\subsection{Star Formation Efficiency}

The SFE, the SF per unit gas mass, decreases with radius from values of $10^{-10} \mathrm{yr}^{-1}$ in the inner ring to $\sim 10^{-11} \mathrm{yr}^{-1}$ in the outskirts. In Figure 5 we show the SFE as a function of radius (normalized to $r_{25}=D_{25} / 2$ ). An increase in the observed scatter is present as a function of radius, from $\sim 0.2$ dex to $\sim 1$ dex.
For comparison, we have plotted in Figure 5 the derived fit for the SFE as a function of radius from Leroy et al. (2008):

$$
\mathrm{SFE}=4.3 \times 10^{-10} \mathrm{yr}^{-1}, \quad \text { if } r<0.4 r_{25} ;
$$

$$
\mathrm{SFE}=2.2 \times 10^{-9} \exp \left(-r /\left(0.25 r_{25}\right)\right) \mathrm{yr}^{-1}, \quad \text { if } r>0.4 r_{25} .
$$

Note that their calibration is valid up to $1.2 r_{25}$ (Leroy et al. 2008).

The agreement between this fit from Leroy et al. (2008) and our SFE radial profile is reasonably good at $0.4 r_{25}<r<1.5 r_{25}$. Although our fit is slightly offset toward higher values, the slope is essentially the same in this radius range. The fit to our data and that of Leroy et al. (2008) are shown in Figure 5 as dashed lines.

From $r>1.5 r_{25}$, the fit by Leroy et al. (2008) would start to deviate considerably from our data, in the same manner as pointed out in other spiral galaxies by Bigiel et al. (2010b). It seems to be more constant, at least for those regions where $\Sigma_{\text {SFR }}$ and $\Sigma_{\text {gas }}$ are above the noise level (blue data points). 


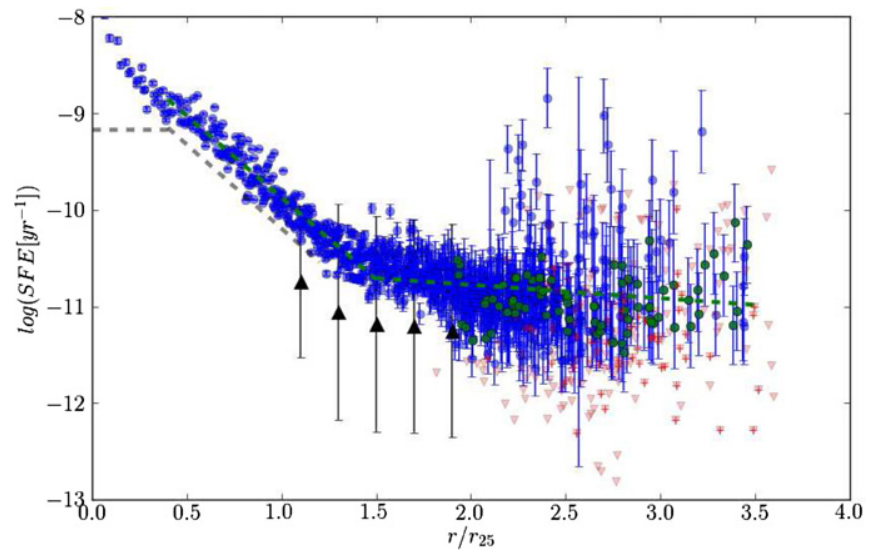

Figure 5. Star formation efficiency (SFE, in logarithmic scale) as a function of galactocentric radius normalized by $r_{25}$, up to $r / r_{25}=3.5$. Blue data points indicate regions whose $\Sigma_{\text {gas }}$ and $\Sigma_{\text {SFR }}$ both are above their noise levels, as in Figure 4. There is an apparent break in the SFE fit at around $r / r_{25}=1.5$. The green dashed line represents a fit to the detected data points: intercept $=-8.2 \pm$ 0.02 , slope $=-1.69 \pm 0.02$ if $r / r_{25}<1.5$ and intercept $=-10.5 \pm 0.1$, slope $=$ $-0.14 \pm 0.05$ if $r / r_{25} \geqslant 1.5$. The gray dashed line shows the fit obtained by Leroy et al. (2008) for 18 nearby galaxies (see Section 3.4). Below $r / r_{25}<0.4$, the gas surface density is likely dominated by molecular gas and the correlation to the fit does not hold. Red data points correspond to regions whose $\Sigma_{\text {gas }}$ is above the noise level but $\Sigma_{\text {SFR }}$ is an upper limit. Green circles represent the detected data points in the kinematically detached region in the SW (Espada et al. 2005). The black triangles represent the median data points from Bigiel et al. (2010b) for a sample of spiral galaxies.

For such regions the $\mathrm{SFE} \simeq 10^{-11} \mathrm{yr}^{-1}$. The fit to our data is shown in Figure 5 as a green dashed line. It fits relatively well ( $\mathrm{rms} \sim 0.5 \mathrm{dex}$ ) over a radius range of $1.5 r_{25}<r<3.5 r_{25}$. Note that the scatter is large enough to consider the fit parameters as estimates only. This region corresponds to the pseudo-ring feature. Many of the detected regions in the radius range $1.7 r_{25}<$ $r<3.5 r_{25}$ are within the kinematically detached clump located to the SW (green circles; Espada et al. 2005).

Red triangles in Figure 5 correspond to upper limits in $\Sigma_{\mathrm{SFR}}$, although detected in $\Sigma_{\text {gas }}$, and thus upper limits in SFE. These data points would probably increase the scatter of SFE at large radii.

\section{MODEL OF THE MULTI-WAVELENGTH LIGHT DISTRIBUTION OF CIG 96}

We inspect in this section whether the photometric properties, including the XUV emission, are common in a spiral galaxy disk (with a given spin and rotational velocity), or whether on the contrary it is a peculiar object not only for its photometric properties but also for its SF history.

\subsection{Model}

We derived photometric radial profiles for CIG 96 not only in the GALEX FUV and NUV bands, but also in the Sloan Digital Sky Survey (SDSS) ugriz bands and the Spitzer/IRAC 3.6 (Werner et al. 2004) and $4.5 \mu \mathrm{m}$ bands (Fazio et al. 2004). ${ }^{13}$

These multi-wavelength radial profiles were fitted with the models of Boissier \& Prantzos $(1999,2000)$ and the IMF from Kroupa (2001), which are able to predict the radial variation of several galactic properties as a function of two parameters: the spin $\lambda$ (non-dimensional specific angular momentum) and

\footnotetext{
13 We used the basic calibrated data publicly available in the Spitzer archive. These images were obtained as part of the Spitzer Survey of Stellar Structure in Galaxies (PI: K. Sheth).
}

the asymptotic velocity of the rotation curve $V_{C}$. Galaxies are modeled as a set of independently evolving rings, with the gas infall timescale depending on both the total galaxy mass and the local mass surface density. Gas is then converted into stars following a KS law, modulated by a dynamical term that mimics the periodic passage of spiral arms (see also Boissier et al. 2003b). Newly born stars follow a user-specified IMF (here we rely on that of Kroupa 2001). Stars of different masses die at different rates, enriching the ISM with metals that are incorporated in subsequent generations of stars. The lifetimes, yields, evolutionary paths, and spectra of stars at different radii are computed as a function of the local metallicity. An initial model was first calibrated to reproduce observables in the Milky Way (MW; Boissier \& Prantzos 1999). This model was then generalized to other galaxies (Boissier \& Prantzos 2000) using the scaling laws derived from the $\Lambda$-cold dark matter scenario (Mo et al. 1998).

The GALEX, SDSS, and Spitzer photometric profiles were simultaneously fitted with these disk evolution models. The internal extinction profiles in the optical and NIR bands were derived from the UV ones using an MW extinction law and a sandwich dust-to-stars geometry (further details on the fitting procedure can be found in Muñoz-Mateos et al. 2011). By minimizing the $\chi^{2}$ between the observed and predicted photometric profiles, the best-fitting values were found to be $\lambda=0.048_{0.012}^{+0.013}$ and $V_{C}=161_{-9}^{+12} \mathrm{~km} \mathrm{~s}^{-1}$. Using other models characterized by an IMF with less massive stars (i.e., Kroupa et al. 1993) or excluding the XUV disk further out than $150^{\prime \prime}$ does not successfully reproduce both photometric profiles and the observed $\mathrm{H}$ I rotation curve, as the ones used here do (Espada et al. 2005).

We plot in each panel of Figure 6 the deprojected surface density profile for each band considered, as well as the fits from the model. The agreement is good for all components. For UV bands, the azimuthally averaged $\mathrm{AB}$ magnitudes seem to be slightly underestimated in the $r=10^{\prime \prime}-15^{\prime \prime}$ region, although in general they agree well.

The KS law was expressed in Section 3.3 in the form $\Sigma_{\mathrm{SFR}}$ $\propto \Sigma_{\text {gas }}^{N}$, with $N$ varying for different annuli. On the other hand, the SFR in the model is expressed as $\Sigma_{\mathrm{SFR}} \propto \Sigma_{\text {gas }}^{N} V(R) / R$. To check the consistency between both definitions, in Figure 4 (bottom) we plot the temporal evolution (from 0 to $13.5 \mathrm{Gyr}$ ) of $\Sigma_{\text {SFR }}$ and $\Sigma_{\text {gas }}$ using the model, and for different radii, 5.5 (blue), 10.9 (green), and $20 \mathrm{kpc}$ (red). No obvious offset is found, which indicates that this galaxy is generally following the SF law implemented in the models, which relies on average main properties of galaxies.

Note that the models used here are not necessarily good at reproducing the properties of galaxy disks out to these large radii. The model itself does not include an imposed threshold, and although it is an extrapolation, it should work reasonably well for low-density regions and outskirts as long as there is no strong threshold effects and the mode of SF does not change. In fact, these models have been tested against observations of low surface brightness galaxies by Boissier et al. (2003a).

\subsection{Predicted Rotation Curve and Gas Surface Density}

The model provides an estimation of the rotation curve and $\Sigma_{\text {gas }}$ as a function of radius. We compare the observed gas surface density with that predicted by the model in the upper panel of Figure 7. A small deviation is found at low radii where most of the gaseous component is in molecular phase, and at large radii from $r>15 \mathrm{kpc}\left(>3^{\prime}\right)$ where the model underestimates the 
CIG $96 \quad \lambda=0.048_{-0.012}^{+0.013} \quad V_{C}=161_{-9}^{+12} \mathrm{~km} / \mathrm{s} \quad \mathrm{K} 01 \mathrm{IMF}$
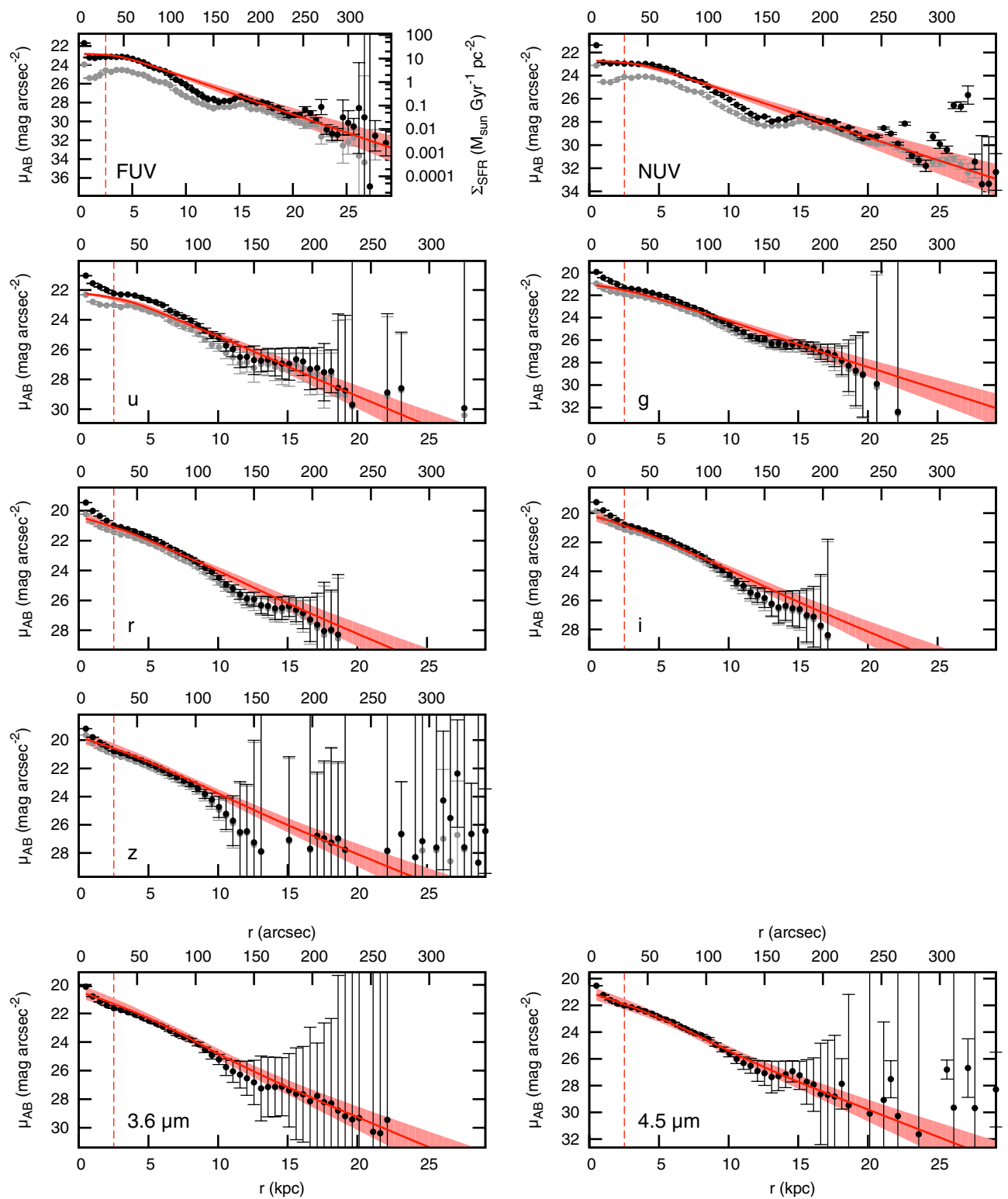

Figure 6. Surface density radial profiles for the different bands: GALEX NUV and FUV, SDSS ugriz bands, and Spitzer 3.6 and $4.5 \mu \mathrm{m}$, in bins of 6". The gray data points are corrected for Galactic extinction and the black data points for internal extinction. The latter are used for the fits using a model based on the IMF by Kroupa (2001, $\lambda=0.048_{-0.012}^{+0.013}$ and $V_{C}=161_{-9}^{+12}$, as explained in Section 4). The best radial profile fits are shown as red solid lines. The red band indicates an estimation of the error, and comprises all the fits within two times the minimum $\chi^{2}$. The red dashed line indicates the radius we use to separate the region of the profile dominated by the bulge (left) and that by the disk (right). Only the latter region is used to perform the fit. A scale conversion for each band to $\Sigma_{\mathrm{SFR}}$ in units of $M_{\odot} \mathrm{yr}^{-1} \mathrm{kpc}^{-2}$ is also shown in the upper left panel.

(A color version of this figure is available in the online journal.)

observed surface density as a result of the external pseudo-ring and the XUV emission in general.

The latter can be probably explained as a result of a recent past event that redistributed part of the gas on a timescale shorter than that probed by the different bands, because they average over the lifetime of the galaxy. UV emission, which as $\mathrm{H}$ I should be sensitive to any perturbation, agrees relatively well to the model. This suggests that a recent event could have occurred, such as accretion, in order for the $\mathrm{HI}$ to show this deviation with respect to the model.

In the lower panel of Figure 7, it can also be seen that the agreement between the observed $\mathrm{H}$ I rotation curve (Espada et al.
2005 ) and that predicted by the model is excellent. Note that the $\mathrm{H}$ I observed rotation curve is not used during the fit.

\section{DISCUSSION AND CONCLUSIONS}

The outer regions of disk galaxies are essential as testing sites for models of disk assembly and evolution. XUV-disk galaxies are ideal laboratories to study low SFR and low gas surface densities conditions. SF in the extreme (low-density, low-metallicity) environment of the outskirts of galaxy disks can elucidate the limiting conditions for gas cloud formation and subsequent $\mathrm{SF}$. 

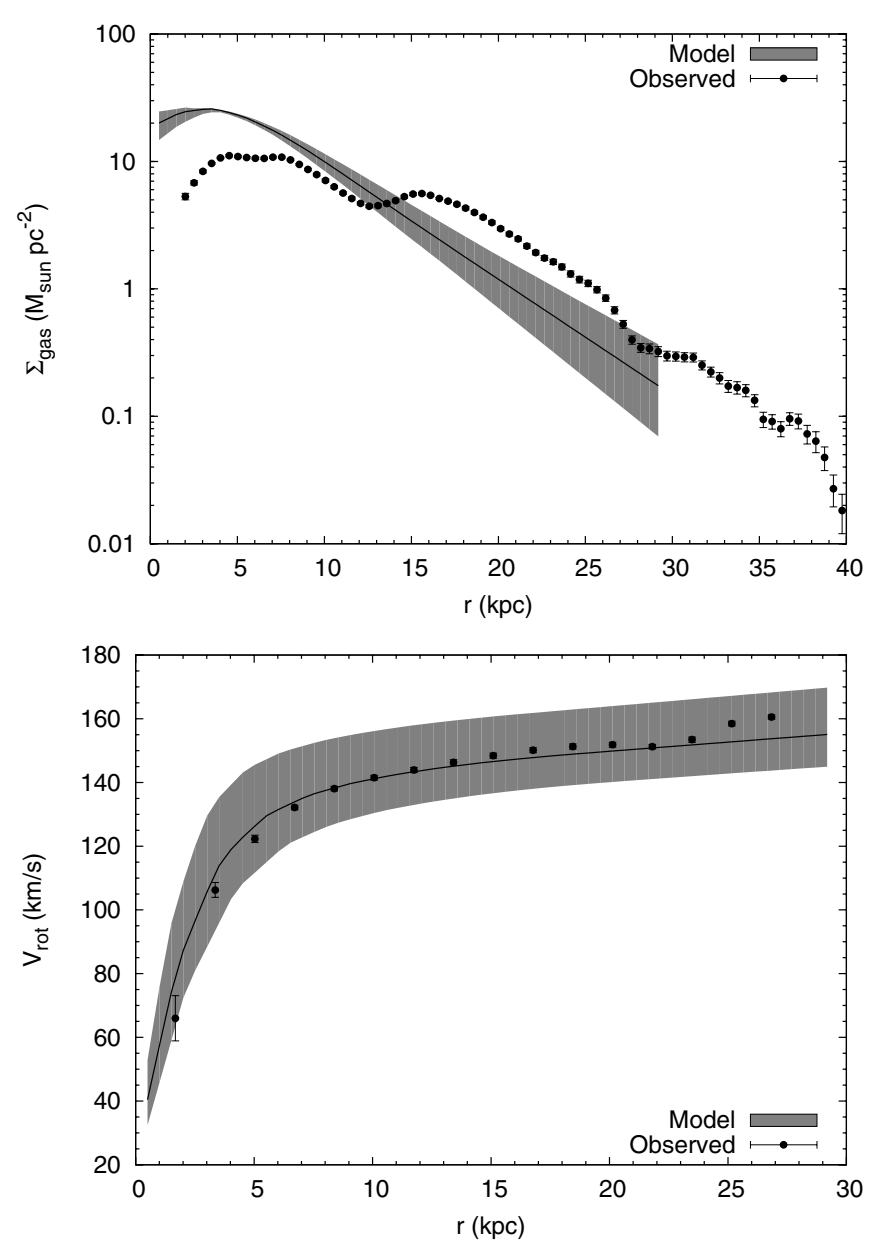

Figure 7. Top panel: observed gas surface density $\left(\Sigma_{\text {gas }}\right)$ radial profile and fit using our model (Section 4) based on the IMF by Kroupa (2001). Bottom panel: comparison of the observed rotation curve (average southern and northern sides; Espada et al. 2005) and that predicted by our model.

CIG 96 is a unique object because it resides in isolation ( $\gtrsim 3$ Gyr since the last major interaction) and has an extended star-forming disk, even though XUV disks are usually ascribed to major interaction events. The observed gas surface density from 2.5 to $4^{\prime} .7$ (15 kpc to $28 \mathrm{kpc}$ ) is larger than that expected from our model, even though the kinematics is on average well reproduced. This suggests that this extra gas and SF could be an anomaly in a (until recently) well behaved disk with a (nondimensional) specific angular momentum of $\lambda=0.048_{-0.012}^{+0.013}$ and an asymptotic velocity of the rotation curve $V_{C}=161_{-9}^{+12} \mathrm{~km} \mathrm{~s}^{-1}$.

Our sensitive and high-resolution data allowed us to derive the power-law index of the KS law locally for different annuli, even at large radii. We find that the slope decreases with radius, being $N \simeq 3.0 \pm 0.3$ in the inner disk $(0.8-1.7)$ and decreasing to $N=1.60 \pm 0.5$ in the outskirts of the gaseous disk (3'3-4.2). The KS law index tends to unity as radius increases. This might reveal a privileged relation between $\mathrm{H} \mathrm{I}$ and $\mathrm{SF}$ where the amount of molecular gas content traced by $\mathrm{CO}$ is small. H i thus could be a good tracer of star-forming gas in these low-density, lowmetallicity conditions. More sensitive observations of both $\mathrm{H}$ I and UV are necessary to reveal what is the limit for the KS index in extended disks.

We found that up to the pseudo-ring feature the SFE radial profile is typical of other disk galaxies, with SFE decreasing with radius. Overall, the SFE spans typical values from $\sim 10^{-9} \mathrm{yr}^{-1}$ to $10^{-12} \mathrm{yr}^{-1}$. Interestingly, the outer part of the $\mathrm{H}$ I disk, the pseudo-ring feature, shows a break in the decreasing trend of the SFE presented, for example, by Leroy et al. (2008). The SFE for regions outside the inner disk $\left(r>1.5 r_{25}\right)$ is SFE $\sim 10^{-11} \mathrm{yr}^{-1}$ (Section 3.3). There are regions that share this constant value up to $3.5 r_{25}$, especially the southern part, which Espada et al. (2005) showed is actually a kinematically detached region from the rest of the disk. Regions with no relevant $\Sigma_{\text {SFR }}$ but above the noise level of $\Sigma_{\text {gas }}$ suggest very low SFE, of the order of about $10^{-11} \mathrm{yr}^{-1}$. The depletion timescales involved are quite large and thus the gas is very likely a reservoir for future SF. Even for the pseudo-ring feature that has a larger SFE for its radius, if $1 \%$ of the gas turns into stars in $10^{8} \mathrm{yr}$, the timescale for consuming all the gas would be large, of the order of $\sim 10 \mathrm{Gyr}$.

Are these deviations in SFE at large radii the rule more than the exception in other XUV disks? There are two pieces of evidence that seem to show that these deviations from normal values may be commonly present in other galaxies. In Figure 1 of Leroy et al. (2008), where SFE is plotted versus galactocentric radius, the average SFE for all the data points is larger than their fit for $r / r_{25}>1.0$. Unfortunately, their calibration limit is $r / r_{25}=1.2$. Probably the most relevant case to compare the SFE at large radii is that of M83. M83's extended H I disk reaches, as in CIG 96, a radius $r \sim 3.5 r_{25}$, and it has been widely studied in the UV and H i by Bigiel et al. (2010a). The SFE in both cases levels off at $r \sim 1.5 r_{25}$ with a value of $10^{-11} \mathrm{yr}^{-1}$. Note that for M83 this corresponds to the depletion time $\tau_{\mathrm{dep}}=10^{2} \mathrm{Gyr}$ (in the right panel of Figure 4 of Bigiel et al. 2010a), also with a scatter $0.5-1$ dex. This evidence suggests that we might have higher SFE in extended disks with respect to that extrapolated from inner radii. In order to check the generality of this result, deeper H I and UV observations of more XUV disks are needed.

The next question is, what mechanism is enhancing the $\mathrm{H}$ I densities and triggering the SF in the outskirts of these galaxies? The isolation of CIG 96 since about $\sim 3$ Gyr ago allows us to discard the major interaction event scenario. Thus, if gravitational instabilities induced by interactions (e.g., Gil de Paz et al. 2005) are producing the observed spiral arms and pseudo-ring feature, then we can reject the major interaction event scenario. It must be due to minor companions (hypothesis explored by Espada et al. 2005), extragalactic clouds, or dark matter subhalos (Tenorio-Tagle 1981; Bekki \& Chiba 2006). Espada et al. (2005) suggested the possibility of an accretion of an HI-rich companion to be responsible for the SW kinematically detached clump and the overall outer ring-like structure.

However, given the remarkable spiral symmetry found in the UV and Hi maps (i.e., two symmetric spiral arms from the $\mathrm{N}$ and $\mathrm{S}$ of the optical disk, and connecting the outer pseudo-ring feature to the $\mathrm{E}$ and $\mathrm{W}$, respectively), one may think that it is an intrinsic property of the galaxy rather than induced by the environment. The outer spiral arms could be due to an instability or could be driven, either by a non-axisymmetric halo (e.g., Machado \& Athanassoula 2010), or by a non-axisymmetric disk. We favor this last option, although it is difficult to prove or disprove since the major axis of this oval would be more or less aligned with the kinematical major axis. But the global morphology makes this option quite plausible. In particular, the arms emanate symmetrically in the NW and SE, and then fall back at the other side. This is a pattern expected in the manifold theory (Romero-Gómez et al. 2006, 2007; Athanassoula et al. 2009a, 2009b, 2010) if the disk is oval and the manifolds allow the orbits to make a $270^{\circ}$ turn while meeting the opposite arm. 
We thank the anonymous referee for a careful reading and very detailed report, which helped to improve this paper significantly. D.E., A.G.d.P., and L.V.-M. acknowledge support for this work from the GALEX Guest Investigator program under NASA grant NNX09AQ356. D.E. was supported by a Marie Curie International Fellowship within the Sixth European Community Framework Programme (MOIF-CT-200640298). D.E., J.S., and L.V.-M. were partially supported by the DGI Grant AYA2008-06181-C02 and the Junta de Andalucía (Spain) P08-FQM-4205. S.V. was partially supported by Junta de Andalucía Grant FQM108, Spanish MEC Grant AYA-2007-67625-C02-02, and a Juan de la Cierva fellowship. A.G.d.P. and J.C.M.-M. are partially financed by the Spanish Programa Nacional de Astronomía y Astrofísica under grants AyA2006-02358 and AyA2009-10368 and the Consolider-GTC project. A.G.d.P. is also financed by the Spanish Ramón y Cajal program. J.C.M.-M. acknowledges financial support from NASA JPL/Spitzer grant RSA 1374189; he also acknowledges support from the National Radio Astronomy Observatory, which is a facility of the National Science Foundation operated under cooperative agreement by Associated Universities, Inc. GALEX is operated for NASA by the California Institute of Technology under NASA contract NAS5-98034. The Spitzer Space Telescope is operated by the Jet Propulsion Laboratory, California Institute of Technology, under contract with the National Aeronautics and Space Administration. Funding for the SDSS and SDSS-II was provided by the Alfred P. Sloan Foundation, the Participating Institutions, the National Science Foundation, the U.S. Department of Energy, the National Aeronautics and Space Administration, the Japanese Monbukagakusho, the Max Planck Society, and the Higher Education Funding Council for England. This research has made use of the NASA/IPAC Extragalactic Database, which is operated by JPL/Caltech, under contract with NASA. We acknowledge the usage of the HyperLeda database (http://leda.univ-lyon1.fr).

Facilities: VLA, GALEX, Sloan, Spitzer

\section{REFERENCES}

Athanassoula, E., Romero-Gómez, M., Bosma, A., \& Masdemont, J. J. 2009a, MNRAS, 400, 1706

Athanassoula, E., Romero-Gómez, M., Bosma, A., \& Masdemont, J. J. 2010, MNRAS, 407, 1433

Athanassoula, E., Romero-Gómez, M., \& Masdemont, J. J. 2009b, MNRAS, 394, 67

Bauermeister, A., Blitz, L., \& Ma, C.-P. 2010, ApJ, 717, 323

Bekki, K., \& Chiba, M. 2006, ApJ, 637, L97

Bettoni, D., Buson, M. L., \& Galletta, G. 2010, A\&A, 519, A72

Bigiel, F., Leroy, A., Seibert, M., Walter, F., Blitz, L., Thilker, D., \& Madore, B. 2010a, ApJ, 720, L31

Bigiel, F., Leroy, A., Walter, F., Blitz, L., Brinks, E., de Blok, W. J. G., \& Madore, B. 2010b, AJ, 140, 1194

Bigiel, F., Leroy, A., Walter, F., Brinks, E., de Blok, W. J. G., Madore, B., \& Thornley, M. D. 2008, AJ, 136, 2846

Boissier, S., Monnier Ragaigne, D., Prantzos, N., van Driel, W., Balkowski, C., \& O'Neil, K. 2003a, MNRAS, 343, 653

Boissier, S., \& Prantzos, N. 1999, MNRAS, 307, 857

Boissier, S., \& Prantzos, N. 2000, MNRAS, 312, 398

Boissier, S., Prantzos, N., Boselli, A., \& Gavazzi, G. 2003b, MNRAS, 346, 1215

Boissier, S., et al. 2007, ApJS, 173, 524

Bosma, A. 1981, AJ, 86, 1791

Bresolin, F., Ryan-Weber, E., Kennicutt, R. C., \& Goddard, Q. 2009, ApJ, 695, 580

Buat, V., et al. 2005, ApJ, 619, L51

Calzetti, D., Kinney, A. L., \& Storchi-Bergmann, T. 1994, ApJ, 429, 582
Cortese, L., Boselli, A., Franzetti, P., Decarli, R., Gavazzi, G., Boissier, S., \& Buat, V. 2008, MNRAS, 386, 1157

de Vaucouleurs, G., de Vaucouleurs, A., Corwin, H. G., Jr., Buta, R. J., Paturel, G., \& Fouque, P. 1991, Third Reference Catalogue of Bright Galaxies, Vol. 1-3 (Berlin: Springer)

Dong, H., Calzetti, D., Regan, M., Thilker, D., Bianchi, L., Meurer, G. R., \& Walter, F. 2008, AJ, 136, 479

Elfhag, T., Booth, R. S., Hoeglund, B., Johansson, L. E. B., \& Sandqvist, A. 1996, A\&AS, 115, 439

Elmegreen, B. G., \& Hunter, D. A. 2006, ApJ, 636, 712

Eskridge, P. B., et al. 2002, ApJS, 143, 73

Espada, D., Bosma, A., Verdes-Montenegro, L., Athanassoula, E., Leon, S., Sulentic, J., \& Yun, M. S. 2005, A\&A, 442, 455

Fazio, G. G., et al. 2004, ApJS, 154, 10

Gil de Paz, A., et al. 2005, ApJ, 627, L29

Gil de Paz, A., et al. 2007, ApJS, 173, 185

Haynes, M. P., van Zee, L., Hogg, D. E., Roberts, M. S., \& Maddalena, R. J. 1998, AJ, 115, 62

Heckman, T., et al. 1995, ApJ, 452, 549

Huchtmeier, W. K., \& Richter, O.-G. 1982, A\&A, 109, 331

Karachentseva, V. E. 1973, Astrofiz. Issled.-Izv. Spets. Astrofiz. Obs., 8, 3

Kennicutt, R. C., Jr. 1998, ApJ, 498, 541

Kennicutt, R. C., Jr., Tamblyn, P., \& Congdon, C. E. 1994, ApJ, 435, 22

Kennicutt, R. C., Jr., et al. 2003, PASP, 115, 928

Kroupa, P. 2001, MNRAS, 322, 231

Kroupa, P., Tout, C. A., \& Gilmore, G. 1993, MNRAS, 262, 545

Leitherer, C., et al. 1999, ApJS, 123, 3

Leon, S., \& Verdes-Montenegro, L. 2003, A\&A, 411, 391

Leroy, A. K., Walter, F., Brinks, E., Bigiel, F., de Blok, W. J. G., Madore, B., \& Thornley, M. D. 2008, AJ, 136, 2782

Machado, R. E. G., \& Athanassoula, E. 2010, MNRAS, 406, 2386

Martin, C. L., \& Kennicutt, R. C., Jr. 2001, ApJ, 555, 301

Martin, D. C., et al. 2005, ApJ, 619, L1

Martini, P., Regan, M. W., Mulchaey, J. S., \& Pogge, R. W. 2003, ApJS, 146, 353

Meurer, G. R., Heckman, T. M., \& Calzetti, D. 1999, ApJ, 521, 64

Mo, H. J., Mao, S., \& White, S. D. M. 1998, MNRAS, 295, 319

Muñoz-Mateos, J. C., Boissier, S., Gil de Paz, A., Zamorano, J., Kennicutt, R. C., Jr., Moustakas, J., Prantzos, N., \& Gallego, J. 2011, ApJ, 731, 10

Muñoz-Mateos, J. C., et al. 2009, ApJ, 701, 1965

Nilson, P. 1973, Uppsala General Catalogue of Galaxies (Uppsala: Astronomiska Observatorium)

Paturel, G., Fang, Y., Petit, C., Garnier, R., \& Rousseau, J. 2000, A\&AS, 146, 19

Paturel, G., Theureau, G., Bottinelli, L., Gouguenheim, L., Coudreau-Durand, N., Hallet, N., \& Petit, C. 2003, A\&A, 412, 57

Prescott, M. K. M., \& Kennicutt, R. C. 2008, in ASP Conf. Ser. 381, Infrared Diagnostics of Galaxy Evolution, ed. R.-R. Chary, H. I. Teplitz, \& K. Sheth (San Francisco, CA: ASP), 181

Romero-Gómez, M., Athanassoula, E., Masdemont, J. J., \& García-Gómez, C. 2007, A\&A, 472, 63

Romero-Gómez, M., Masdemont, J. J., Athanassoula, E., \& García-Gómez, C. 2006, A\&A, 453, 39

Salpeter, E. E. 1955, ApJ, 121, 161

Schlegel, D. J., Finkbeiner, D. P., \& Davis, M. 1998, ApJ, 500, 525

Schmidt, M. 1959, ApJ, 129, 243

Speltincx, T., Laurikainen, E., \& Salo, H. 2008, MNRAS, 383, 317

Tenorio-Tagle, G. 1981, A\&A, 94, 338

Thilker, D. A., et al. 2005, ApJ, 619, L79

Thilker, D. A., et al. 2007, ApJS, 173, 538

Thilker, D. A., et al. 2009, Nature, 457, 990

Toomre, A. 1964, ApJ, 139, 1217

Tully, R. B. 1988, Nearby Galaxies Catalog (Cambridge: Cambridge Univ. Press)

Verdes-Montenegro, L., Sulentic, J., Lisenfeld, U., Leon, S., Espada, D., Garcia, E., Sabater, J., \& Verley, S. 2005, A\&A, 436, 443

Verley, S., Corbelli, E., Giovanardi, C., \& Hunt, L. K. 2009, A\&A, 493, 453

Verley, S., Corbelli, E., Giovanardi, C., \& Hunt, L. K. 2010, A\&A, 510, A64

Verley, S., et al. 2007a, A\&A, 470, 505

Verley, S., et al. 2007b, A\&A, 472, 121

Walter, F., Brinks, E., de Blok, W. J. G., Bigiel, F., Kennicutt, R. C., Thornley, M. D., \& Leroy, A. 2008, AJ, 136, 2563

Werner, M. W., et al. 2004, ApJS, 154, 1

Witt, A. N., \& Gordon, K. D. 2000, ApJ, 528, 799

Wong, T., \& Blitz, L. 2002, ApJ, 569, 157 